\title{
Study on ship domain of large vessels in restricted waters based on AIS data
}

\author{
Tianrui Zhou, Zhihui Hu, Jiacai Pan \\ Navigation Institute, Jimei university,Xiamen 361021,China. \\ 1241514977@qq.com
}

Keyword. AIS data, Ship domain, Restricted waters, large vessels.

\begin{abstract}
In order to obtain the ship domain restricted waters, this paper established a model of the method by massive AIS data. By selecting the ship's AIS data, counting the distance and relative orientation between the surround ship and the target ship. Considering the ship size, the vicinity of the target ship is divided into several fan-shaped areas, Calculated the number of times in each area and a single vessel superposition diagram was obtained, composite same single vessel superposition diagram into the multi-ship superposition chart. Finally, calculated the density of each region, and determining the boundary of ship domain by use mean maximum density. The method is validated by using AIS data from Xiamen port, studying four ship domain, the target ship length is above 200 meters and surround ship length in $0 \sim 100,100 \sim 200,200 \sim 300$ and more than 300 meters, the results show that the size of ship domain are increasing along the ship length.
\end{abstract}

\section{Introduction}

The ship domain is refers to avoid a collision,keep an active area in order to other vessel don't encroach the area [1], It often used to measure collision risk when the ship navigating at the port or narrow waterway. Since 1960s, Fujii put forward the concept of ship domain , the experts and scholars in the field have never stopped. the British scholar Coodwin statistics traffic flow on the Southern Beihai waters, constructed ship domain of open water combine to the international regulations for preventing collisions at sea, the model have three fan-shaped sectors by the port, starboard part and the rear part [2]. Davis considering the Goodwin model having much defects such as the boundary discontinuity, difficult to simulate, By smoothing the boundary of the ship, a circular ship field for simulation and application is proposed, pointed out that the target ship is located at the lower left of the ship model center[3]. Pietrzykowski established a non fixed ship domain by statistical means[4]. Smierzchalski proposed a hexagonal ship domain model, the size is determined by the vessel of speed, gyro parameters, and so on[5]. Jia chuanying considered the speed and size of vessel, established the size of domian,relationship between length and speed, and put forward the variable size of domain in crowded field of water[6].Chen houzhong proposed a two-dimensional ship domain model for confined waters of bridge area,Combining the water carrying capacity of bridge area[7]. Huang Yin established dynamic ship domain model of inland river under the influence of wind, combining to the characteristics of inland waterway and navigation, and considering the influence of air flow on the ship's domain[8].

The above analysis methods can be divided into statistical analysis method and analytic method [9]. The accuracy of observational data from statistical analysis is poor. The analytic domain of ship enables quantification of domain boundaries, and considering the ship maneuvering performance, compared with the statistical domain model, the ship behavior of ability occupy a dominant position, but did not consider the human and environmental factors in the field of the model, so the model can't meet the complex factors of ship domain environment or demand, the application is not high.

In recent years, scholars have carried out a series of studies on the ship domain by using intelligent technology. Wang proposed four- dimensional ship domain model based on the analytical framework, improve flexibility and maneuverability, combined with fuzzy system theory, put 
forward fuzzy four- dimensional ship domain mode[10]. Pietrzykowski proposed dynamic fuzzy model of ship domain by using artificial neural network, the distance and relative orientation between two vessels, to ship heading and other variables as input, the vessel navigation risk as output, get relation between input and output and determine the boundary of fuzzy ship domain, but it does not take into account environmental factors[11]. Hansen used the AIS data from Fehmarn Belt between Denmark and Germany, obtained the water of ship domain by using superimposed methods[12], but without considering the influence of ship size on the ship domain. In confined waters, the size and geometry of ships have a greater impact on the ship's domain [13-15], so this paper studies the large ships of ship domain.

AIS data is automatically acquired by the automatic ship identification systems,it contains a large of information in the waters,such as the vessel location(latitude,longitude), navigation status(heading,

Course over the ground), ship size,etc. Compared to the same conditions, the information from radar data only contains basic ship position and heading, can not get the size of ships, etc. the information is much richer. Therefore, in order to accurately calculate the ship domain in restricted waters, it should be build model of ship domain in restricted waters by using AIS data. Firstly, filtering those required ship information by using massive AIS data, as the target ship sailing and calculating the distance and the azimuth of the surround ship relative to the target ship, a single vessel superimposed diagram is formed, then sorting and stacking the single ship overlay diagram according to the size of the ship,get the specific type Ship stacking chart, Ship stacking chart is divided into several sub region, counting ship density of each region, and finally obtain ship domain in restricted waters.

\section{Algorithm}

\section{Definition}

The ship domain in restricted waters is determined by the relative position from ship's encounter state, and the relative position consists of distance and relative orientation which surround vessel relative to the target ship. Firstly, we statistical ship distribution near 3 nautical miles around target ship by use of massive AIS data,due to the distribution of a single ship is not complete, so it's necessary to superimposed same type distribution of a single ship, then the target near area is divided into a number of small fan-shaped intervals, the radial direction within the target ship near 3 miles is divided into several concentric circular interval, these small fan-shaped and concentric circle cross composed of several small area, as shown in figure 1

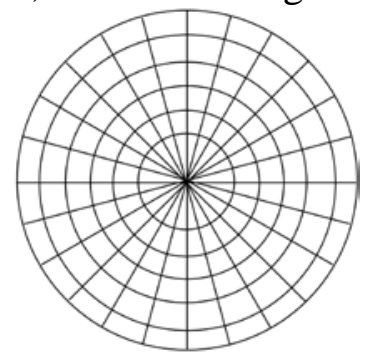

FIGURE 1. District partition

We calculate the number of vessel $Q_{i j}$ and $A_{i j}$ area in each region,then vessel density of each small area can be obtained by the ratio, formula is as follows[16]

$$
D_{i j}=Q_{i j} / A_{i j}
$$

The terms used in this article are as follows

AIS data: receiving by ship automatic identification system, which contains the position of specific ship, navigation condition and ship size in continuous time.

Target ship: the vessel to be analyzed for navigation more than a period of time in restricted waters. 
surround ship: all vessels in 3 nautical miles around target vessel center.

Single ship distribution diagram:the distribution of ship around target ship at a given moment.

Single vessel overlay diagram: the superposition of ships around the target ship over time.

Multi vessel superposition charts: single ship graphs superimposed for same type.

Average maximum density: the average density of the maximum density for all radial fan regions.

Ship superposition chart of particular type: we classify target ship and surround ship in a standard (this paper to classify the ship with the length range), superimposed on the distribution of ship which meet conditions, so as to obtain a specific type of ship overlay.

\section{The principle of algorithm}

The principle of algorithm:firstly,the AIS data was processed, a number of target ships are selected, the target ship is taken as the center, and the distribution of the ship around the target ship at each moment is calculated, as the target ship continues to sail, the distribution of ships around each moment is superimposed to obtain single ship overlay chart, the particular type of single ship overlay graph is superimposed to obtain particular type of multi ship superimposed diagram, the algorithm flow chart as shown figure 1.2

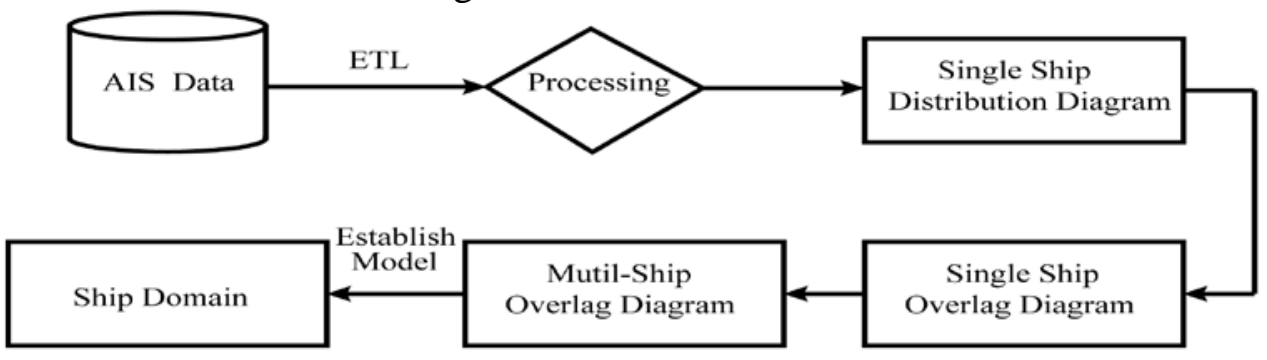

FIGURE 2. The model of ship dynamic domain

\section{The method of calculating the ship relative position}

Step1: The AIS data processing: the main work is delete the Maritime Mobile Service Identity (Maritime Mobile Service Identity, MMSI)) for 0 records; remove obvious error in the AIS data such as velocity $>100$, longitude $>180$, latitude $>90$, heading $>360$, the length and width is obviously does not meet the requirements.

Step 2: Target ship is selected: from the AIS data, we select those ship which speed and course are stabilize and navigating over a certain length of time.

Step 3: surround ship is selected: the target ship as the center, we selecting those vessel which time difference $\Delta \mathrm{t}\langle\mathrm{t}$, because most of the AIS data broadcast time interval in $2 \mathrm{~S} \sim 3 \mathrm{~min}$, so this paper $\mathrm{t}$ is take for $3 \mathrm{~min}$, when there is more than a moment in t time,we select shortest time away from the center of the time.

Step 4: The distribution of surround ships: taking the target ship as center, we calculate relative position of the ship around target ship at any given moment.Relative positions are made up of distance and relative orientation. Because the AIS data of each ship broadcast time interval is not consistent, so the surround vessel is converted to the same time as the target ship when we are calculate the relative position, the conversion formula as follows;

$$
\begin{gathered}
V_{A 1}=V_{A 0}+\frac{t_{1}-t_{0}}{t_{2}-t_{0}}\left(V_{A 2}-V_{A 0}\right) \\
A_{1}=A_{0}+\frac{\left(V_{A 0}+V_{A 1}\right)\left(t_{1}-t_{0}\right)}{\left(V_{A 0}+V_{A 2}\right)\left(t_{2}-t_{0}\right)}\left(A_{2}-A_{0}\right)
\end{gathered}
$$

Where $t_{1}$ is a time of the target ship, $t_{0}, t_{2}$ is the closest two time of surround ship to target ship. $V_{A 1}$ is speed of the ship alongside target ship at the time $t_{1}, V_{A 0}, V_{A 2}$ is the time $t_{0}, t_{2}$ corresponding speed, $\mathrm{A}$ is the position of surround ship. 
Step 5: Calculates the relative position of the surround ship to target ship. The relative positions include the distance $\mathrm{D}$ and relative azimuth $\mathrm{B}$.

The distance $\mathrm{D}$ is calculated as follows;

two vessel of latitude,longitude is known, respectively is A and B, it is mean target ship and

surround ship,A point is $\left(\varphi_{1}, \lambda_{1}\right)$, B point is $\left(\varphi_{2}, \lambda_{2}\right), D_{i}$ was the difference longitude between two vessel.

$$
\begin{aligned}
& \cos \left(D_{1}\right)=\sin \left(\varphi_{1}\right) \times \sin \left(\varphi_{2}\right)+\cos \left(\varphi_{1}\right) \times \cos \left(\varphi_{2}\right) \times \cos \left(D_{\lambda}\right) \\
& \tan \left(C_{1}\right)=\sin \left(D_{\lambda}\right) \div\left(\cos \left(\varphi_{1}\right) \times \tan \left(\varphi_{2}\right)-\sin \left(\varphi_{1}\right) \times \cos \left(D_{\lambda}\right)\right)
\end{aligned}
$$

$D_{1}$ is air-range, $C_{1}$ is azimuth, A point of latitude in both north and South should be taken positive, $\mathrm{B}$ point of latitude is taken positive when its latitude is same to $\mathrm{B}$, or is taken negative. $D=D_{1} \times 60$, $\mathrm{D}$ is distance,the result $C_{1}$ is half circle method, the first letter of name as same to the A point latitude, the second letter as the different longitude $D_{\imath}$, So need to conversion.

If $C_{1}$ is negative, then $C=C_{1}+180$

If A point of latitude is north; if $D_{\lambda}=\lambda_{2}-\lambda_{1}>0, C=C_{1}$, if $D_{\lambda}=\lambda_{2}-\lambda_{1}<0$, then $C=360-C_{1}$, if A point of latitude is south; if $D_{\lambda}=\lambda_{2}-\lambda_{1}>0, C=180-C_{1}, D_{\lambda}=\lambda_{2}-\lambda_{1}<0$, then $C=180+C_{1}$

After getting the final course $C$, according $B_{1}=C-H D G$, HDG is the heading of target ship, because some ships don't install magnetic compass,so HDG instead by course over Ground COG. if $B_{1}>0$, then $B=B_{1}$, if $B_{1}<0, B=B_{1}+360$.

\section{The traffic flow analysis of Xiamen port}

After selecting the incomplete information and error information in AIS data, the latitude and longitude range is chosen as $24.3167 \mathrm{~N} \sim 24.4324 \mathrm{~N}, 118.0705 \mathrm{E} \sim 118.1966 \mathrm{E}$, the time is April , May, June in 2005. We establish SQL database of Xiamen port in restrict waters, we are obtain distribution of ship length by statistical analysis of AIS data,the distribution of ship length as shows

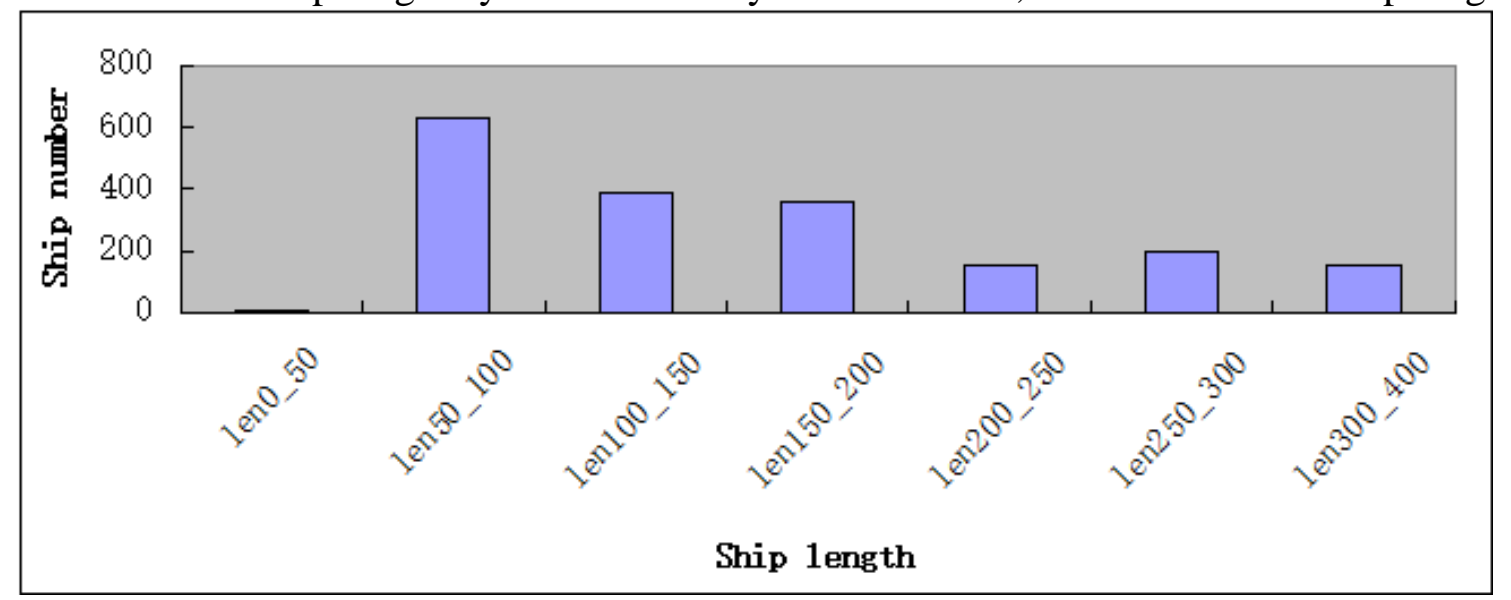

FIGURE 3. The distribution of ship length from Xia men

From the diagram, we can find the length scope of vessel is mainly distribute between $50 \sim 400$ meters, so the length of vessel is divided into four grades ,there are $0 \sim 100,100 \sim 200,200 \sim 300$, more than 300 meters.

\section{The calculation of ship domain in restricted waters of Xiamen port}

\section{A method of determining the boundary of ship domain by mean maximum Density}

We are take the target ship's bow of 0 degrees to the center, rotate clockwise at 5 degrees intervals, divide the azimuth into 72 sectors, and divide the distance into 60 concentric circles within 3 nautical miles at 0.05 nautical miles interval, These concentric circles and cross sector 
were composed of 4320 regions. We composite same type of distribute position of surround vessel, then calculate the density in each area.

Reference to the fields of thought Fujii, at the same time avoid influence of excessive vessel density caused by non avoidance action, we use the average maximum density decide to the boundary of ship domain. We find the first coordinates of the center point which the density of each radial fan region equal to the mean maximum density, then we are handle the 72 points by Matlab software to get fitting curve, so as to obtain the boundary of ship domain.

We handle the export of 72 points fitting ellipse by using least square method,fitting formula as follow;

$$
\begin{aligned}
& F=@(p, x) p(1) * x(:, 1) .^{2}+p(2) * x(:, 1) * x(:, 2)+ \\
& p(3) * x(:, 2) .^{2}+p(4) * x(:, 1)+p(5) * x(:, 2)+p(6)
\end{aligned}
$$

In this paper, we are study on the target ships above 200 meters,surround ship of length in $0 \sim 100,100 \sim 200,200 \sim 300,300 \sim 400$, the fitting curves are a, b,c,d.in figure3. According to the results, $0 \sim 100$ meter ship domain length of long axis is equal to 0.4116 nautical mile, the length of short axis is equal to 0.3766 nautical mile, 100 200 meter ship domain length of long axis is equal to 0.4657 nautical mile, the length of short axis is equal to 0.3809 nautical mile, 200 300 meter ship domain length of long axis is equal to 0.6214 nautical mile, the length of short axis is equal to 0.4836 nautical mile, 300 400 meter ship domain length of long axis is equal to 0.6831 nautical mile,the length of short axis is equal to 0.5126 nautical mile. It can be concluded that the length of long axis are increasing with the increase of ship length.

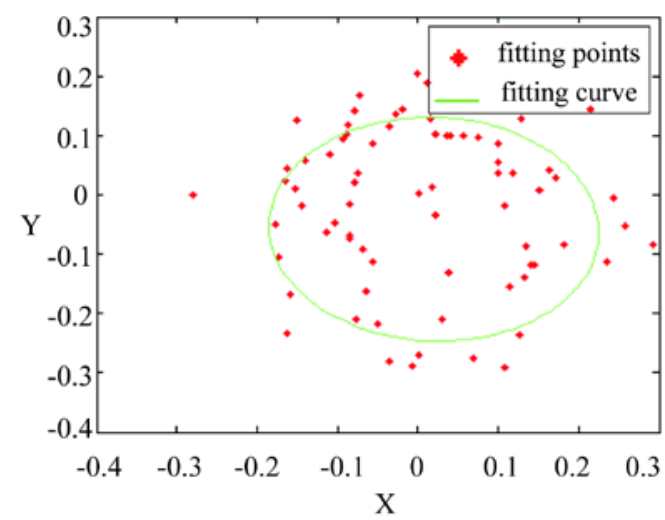

(a)

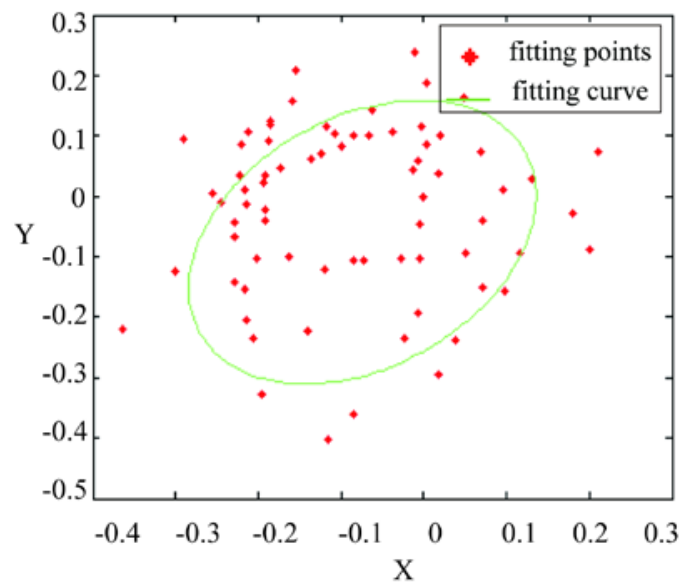

(c)

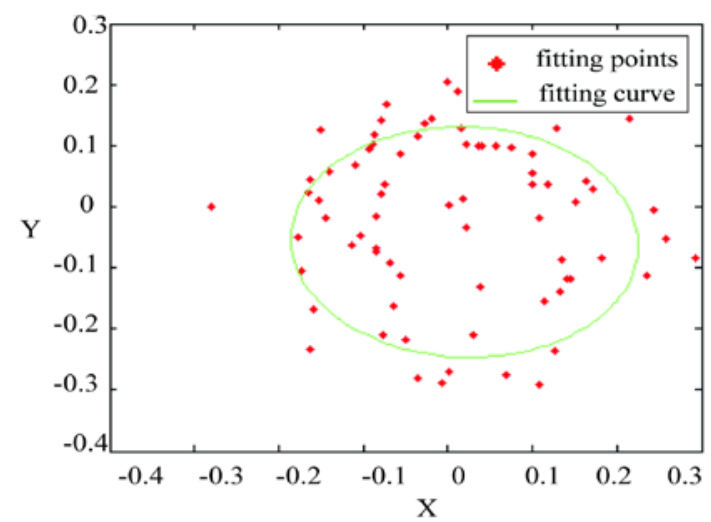

(d)

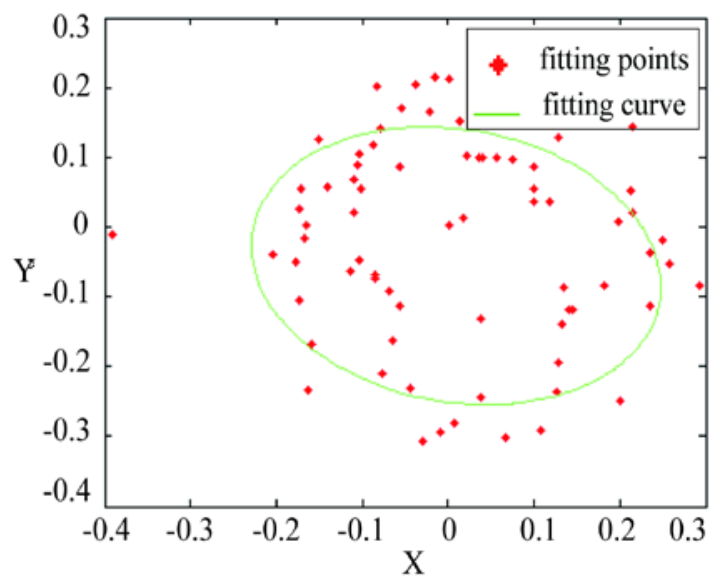

(b)

FIGURE 4. The distribution of ship domain

\section{Conclusion}

This paper put forward method of calculation ship domain in restricted waters.Because the AIS broadcast time is not the same,so we are calculate the relative position need to conversion moment 
of surround ship into target ship,with target ship sailing, we can obtain distribution of single ship, then composite same distribution of single ship according to the length of the ship classification,and we use the method of average maximum density to filing the boundary of ship domain. From the result, we can conclude in the direction of the long axis and short axis length of ship domain are increasing with the length of ship,the range of increased in long axis is greater than the short axis.

\section{Acknowledgments}

This work was financially supported by Fujian Natural Science Foundation of China fund (2015J01214)

\section{References}

[1] Fujii Y, Tanka K. Traffic capacity[J].Journal of navigation,1974,24(4):543-552.

[2] Goodwin E M. A statistical study of ship domains[J].Journal of navigation,1975,28(3):328-344.

[3] Davis P V, DOVE M J , STOCKEL C.T.A computer simulation of marine traffic using domains and arenas[J].Journal of Navigation,1980,33:215-222.

[4] Pietrzykowskz.Ship's fuzzy domain-a criterion for navigational safety in narrow fairways[J].Journal of Navigation,2008,61(3):499-514.

[5] Smierzchalski R, Michalewicz Z. Modeling of ship trajectory in collision situations by an evolutionary algorithm [J].IEEE Transactions on Evolutionary computation, 2000,4(3):227-241.

[6] JIA Chuan Ying. Ship domain in congested water area[J].Journal of Dalian Maritime University,1985,15(4):15-19.

[7] CHEN Hou Zhong,GUO Guo Ping.Research of ship domain and the traffic capacity in paratactic bridge water area[J].Ship and Ocean Engineering,2008,37(5):113-116.

[8] Huang Yin. The model and application of inland ship domain under influence of wind and current [D]. Wuhan university of science and engineering.2012

[9] LIU Shao Man,WANG Ning,WU Zhao-lin.Review of research on ship domain[J].Journal of Dalian Maritime University,2011,37(1):51-54.

[10] Wang Ning .An intelligent spatial collision risk based on the quantification ship domain[J].Journal of Navigation, 2010,63(4):733 -749.

[11] Pietrzykowskz.Ship' s domain-a criterion for navigational safety in narrow fairways[J].Journal of Navigation,2008,61:499-514.

[12] Hansen M G,Jensen T K, Lehn-Schiolen T, et al. Empirical Ship Domain based on AIS Data [J].The Journal of Navigation,2013,66:931-940.

[13] SZLAPCZYNSKI R. A uinfied measure of collision risk derived from the concept of a ship domain[J].Journal of Navigation,2006,59(3):477-490.

[14] Wang Ning, MENG xiao-yao,XU Qing-yang, et al. A unified analytical framework for ship domains[J].Journal of Navigation,2009,62(4):643-655.

[15] Wang Ning. A novel analytical framework for dynamic quaternion ship domains[J].Journal of Navigation,2013,66(2):265-281.

[16] Pan Hui.Analysis of vessel density and distribution.[J]Chinese water transportation,2007.5(3): $17-18$ 